\title{
Échographie au point d'intervention en médecine interne
}

u cours des dernières décennies, diverses percées technologiques ont modifié en profondeur la façon dont nous pratiquons la médecine. Aussi bien dans ses aspects cliniques et que dans son administration, la pratique médicale évolue : il suffit de penser aux endoprothèses qui remplacent les interventions chirurgicales ou aux dossiers numériques qui prennent le relais du papier. Quant à la médecine interne, elle connaîtra bientôt une transformation importante; les internistes devront en effet apprendre à utiliser couramment l'échographie au point d'intervention. Les applications de ce type d'échographie en médecine interne sont innombrables : on peut penser entre autres à l'évaluation précise du pouls jugulaire chez un patient gravement atteint, à la réalisation de ponctions artérielles ou veineuses, ou au diagnostic d'une tamponnade péricardique, d'une embolie pulmonaire ou de la présence de liquides corporels à drainer, pour n'en nommer que quelques-unes.

Certains collègues utilisent déjà cette technologie pendant l'examen clinique ou leurs interventions au chevet des patients, mais pour beaucoup dentre nous, elle ne fait pas encore partie des habiletés de base; cela peut être dû au manque de formation, ou au fait que léquipement n'est pas disponible dans certains milieux cliniques. Mais les choses vont changer, et léchographie au point d'intervention finira par faire partie des compétences obligatoires pour tous les internistes en exercice.
Dans ce numéro de la Revue, Lewis et coll. présentent une évaluation des besoins en échographie au point d'intervention, que ce soit pour répondre à une question clinique ou pour guider une intervention. Comme le Collège royal des médecins et chirurgiens du Canada nexige pas encore de tous les résidents en médecine interne qu'ils suivent une formation structurée en échographie au point d'intervention, les chercheurs sétaient donné pour objectif de mener une évaluation systématique des besoins quant à l'intégration d'une telle formation dans les études de médecine interne. Leurs résultats montrent que certains résidents en médecine interne utilisent déjà léchographie au point d'intervention, mais sans avoir reçu de formation structurée dans ce domaine; on peut se demander quels résultats on obtiendrait auprès des internistes en exercice.

Tous les programmes de résidence finiront par se doter d'une formation en échographie au point d'intervention, mais les internistes en exercice devront eux aussi apprendre à l'utiliser. Il ne faut surtout pas sous-estimer l'importance de cette compétence; le milieu de la médecine interne devra faire preuve de leadership pour permettre à tous les internistes de maîtriser les techniques déchographie au point d'intervention. 